International Journal of Current Microbiology and Applied Sciences

ISSN: 2319-7706 Volume 9 Number 2 (2020)

Journal homepage: http://www.ijcmas.com

Original Research Article

https://doi.org/10.20546/ijcmas.2020.902.098

\title{
Exploitation of Heterosis for Growth and Flowering Traits in kharif Marigold (Tagetes erecta L.)
}

\author{
Chayanika Lahkar*, Reena P. Borkakati and Gargi Sharma \\ Department of Plant Breeding and Genetics, Assam Agricultural University, \\ Jorhat-13, India \\ *Corresponding author
}

\section{Keywords}

Heterosis, Mid parent, Better parent, Standard parent, Tagetes erecta $\mathrm{L}$.

Article Info

Accepted:

08 January 2020

Available Online:

10 February 2020

\section{A B S T R A C T}

A line $\times$ tester set of 13 parents involving 3 male sterile lines and 10 pollen parents of African marigold (Tagetes erecta L.) and their 30 hybrids were analyzed for heterosis with respect to various growth and flowering traits. The hybrids MSC $3 \times$ JS3 and MSC1 $\times$ JS5 exhibited maximum heterosis over mid parent and better parent respectively, for number of branches per plant, whereas MSC1 $\times$ JS1 showed highest heterosis over both mid parent and better parent for plant height. The maximum heterosis value for early flowering were observed in the hybrid, MSC1 $\times$ JS2 over better, mid and standard parent respectively, however the hybrid MSC2 $\times$ JS3 exhibited maximum heterosis over both mid parent and better parent for traits, duration of flowering and plant spread; and MSC1 $\times$ AAUM3 and MSC3 $\times$ Pusa Narangi Gainda reported highest values of heterosis over standard parent for duration of flowering. The hybrids, MSC $3 \times$ Pusa Basanti Gainda showed highest value of average heterosis and MSC3 $\times$ Pusa Narangi Gainda exhibited maximum heterosis over better parent and standard parent, respectively for number of flowers per plant. For flower size, the maximum heterosis over mid, better and standard parent were observed in hybrids, MSC3 $\times$ JS2, MSC2 $\times$ AAUM4 and MSC $3 \times$ AAUM4 respectively. The hybrids, MSC1 $\times$ Pusa Basanti Gainda showed highest value of average heterosis and MSC1 $\times$ AAUM5 exhibited maximum heterosis over better parent and standard parent, respectively for flower yield per plant. The hybrids, MSC3 $\times$ AAUM5, MSC1 $\times$ AAUM5 and MSC3 $\times$ AAUM3 exhibited highest heterosis for vase life of flowers over mid parent, better parent and standard parent, respectively.

\section{Introduction}

Marigold (Tagetes spp.) is one of the most commonly grown flower crops among the ornamentals commercially grown in India. This genus includes 33 species, of which two species i.e., Tagetes erecta L., the African marigold, and Tagetes patula L., the French 
marigold are commonly cultivated in India. The African marigold is the dominant one having high demands for loose flower production in India. Tagetes erecta L., a member of Asteraceae family, is a hardy annual type that bears single to fully double sized flowers. It is native to Mexico. It has wide range of utility from loose flower, garland making, landscape gardening, poultry feed, cosmetics and perfumery, oil and pigment extraction to pharmaceuticals. It also plays a major role in the religious and social ceremonies of India. Marigold is largely known for its antimicrobial, insecticidal, antioxidant, analgesic activity and nematode management properties. Commercially, the importance of marigold is also well established in the markets of Assam. Cultivation of marigold during the kharif season (June-October) is yet to be a successful venture in Assam primarily for want of suitable variety. The mismatch in demand and supply during this season results in high rise in market price, especially during the festive months. Moreover, very few hybrids are available in marigold and a meagre amount of information is available on the breeding aspect of this crop. In this situation heterosis can play a prime role for increasing the quality production of marigold. Heterosis has contributed significantly towards enhanced production of numerous crops and it has become the basis of multibillion dollar agribusiness in the world (Phillips, 1999). Crossing between genetically diverse parents is a commonly adopted method to induce variation than that in existing cultivars in terms of novelty, high yield and increased quality of flower. Recently, attempts were made with considerable success in developing marigold hybrids. Very less work so far has been achieved on genetic improvement of Tagetes spp. through hybridization in India. Considering the fact, there is a need for collection, multiplication, preservation and utilization of this natural wealth. Keeping all the above points into consideration, in order to contribute towards improvement in marigold with respect to enhancement in growth and flowering traits, the present investigation was carried out with the objective to study the magnitude of heterosis in $30 \mathrm{~F}_{1}$ hybrids for different vegetative, flowering, yield, vase life and floriculturally important parameters.

\section{Materials and Methods}

The experimental materials comprised of 13 genotypes of African marigold, which included three male sterile lines namely, MSC1, MSC2 and MSC3 and a set of ten diverse pollinators/ testers namely, Pusa Narangi Gainda (PNG), Pusa Basanti Gainda (PBG), AAUM3, AAUM4, AAUM5, JS1, JS2, JS3, JS4 and JS5. All these genotypes were grown and maintained in the Instructional-Cum-Research (ICR) Farm of Assam Agricultural University, Jorhat (Assam). The field trials including crossing was done in the rabi season, 2017-18 for the production of $\mathrm{F}_{1}$ hybrid seeds. The crossing of 3 male sterile lines and the 10 testers was done in line $\mathrm{x}$ tester mating design to obtain $30 \mathrm{~F}_{1}$ hybrid combinations. The hybrids along with the parental lines were planted in Randomized Block Design (RBD) with three replications having a spacing of $45 \mathrm{~cm} \times 45 \mathrm{~cm}$ for their evaluation in kharif season, 2018. All the recommended cultural practices were followed throughout the growth period of the crop. The data of randomly selected five plants from each treatment over replication were recorded for all the characters viz., number of branches per plant, plant height $(\mathrm{cm})$, days to $50 \%$ flowering, plant spread $(\mathrm{cm})$, duration of flowering (days), number of flowers per plant, flower weight (g), flower size $(\mathrm{cm})$, flower yield per plant $(\mathrm{g})$, vase life (days), at appropriate stages of the plant growth. The data were statistically analyzed 
for assessment of mean performance of the genotypes with respect to yield and yield attributing traits and the exploitation of heterosis of the cross combinations following Line $\mathrm{x}$ Tester analysis developed by Kempthorne (1957). In the present study, attempt had been made to know the magnitude of heterosis, percentage increase or decrease of $F_{1}$ hybrids over mid parent (average heterosis/MP), better parent (heterobeltiosis/BP) and standard heterosis (SH) of thirty crosses for the different growth and flowering characters in desirable direction (Turner 1953, Hays et al., 1955). For estimating the magnitude of standard heterosis $(\mathrm{SH})$, the performance of $\mathrm{F}_{1}$ hybrids were compared with the line, MSC2.

The field trials were conducted during rabi (October-February), 2017-18 and kharif (June-October), 2018 for the production of $F_{1}$ hybrid seeds and their evaluation, respectively, at the Instructional-CumResearch (ICR) Farm of Assam Agricultural University, Jorhat. The materials utilized for the study consisted of thirteen varieties of African marigold (Tagetes erecta L.), out of which three were used as lines namely, MSC1, MSC2, MSC3 and ten were used as testers namely, Pusa Narangi Gainda (PNG), Pusa Basanti Gainda (PBG), AAUM3, AAUM4, AAUM5, JS1, JS2, JS3, JS4 and JS5. The crossing was performed in the rabi season as per Line $\mathrm{x}$ Tester mating design given by Kempthorne (1957) and thirty crosses were generated. All statistical analysis were performed using WINDOSTAT version 8.6 (statistical software developed by Indostat Services, Hyderabad) licensed to LAN Indian Institute of Horticultural Research, Hessaraghatta, Bangalore. Data were uniformly recorded and subjected to analysis of variance (Singh and Chaudhary, 1985).

Mid-parent heterosis (MPH), better-parent heterosis $(\mathrm{BPH})$ and standard heterosis $(\mathrm{SH})$ for each cross were calculated as percentage deviation from the mid-parent (MP), betterparent (BP) and standard parent (SP) values, respectively (Turner, 1953; Hays et al., 1955).

Mid Parent Heterosis, $M P H=\frac{F 1-M P}{M P} \times 100$

Better Parent Heterosis, $B P H=\frac{F 1-B P}{B P} \times 100$

Standard Heterosis, $S H=\frac{F 1-S P}{S P} \times 100$

Where,

P1, P2, F1, MP, BP, SP denotes the mean values of parent-1, parent-2, $\mathrm{F}_{1}$, mid parent, better parent and standard parent, respectively.

\section{Results and Discussion}

\section{Estimation of magnitude of heterosis}

The invigoration of progress in crop improvement by plant breeding was induced by an appropriate exploitation of heterosis. Hybrids in marigold have earned more importance today due of their remarkable ability in improving production. The results of the study indicated that both the nature and magnitude of heterosis vary from trait to trait depending upon the cross combinations and considerable heterosis was observed in both positive and negative directions. The extent of heterosis had been measured as heterosis over mid parent, better parent and standard parent. Heterosis of $30 \mathrm{~F}_{1}$ hybrids for growth and flowering related traits were studied.

More number of branches per plant is one of the major parameters which can support yield and its component traits. The trait of branch number has an indirect relation in enhancement of flower production, so positive heterosis is desirable for this trait. For number of branches per plant, the range 
of heterosis varied from $-30.10 \%$ (MSC2 $\times$ JS1) to $20.22 \%($ MSC1 $\times$ JS5) over better parent, $-21.90 \%$ (MSC3 $\times$ PBG) to $29.26 \%$ $(\mathrm{MSC} 3 \times \mathrm{JS} 3)$ over mid parent and $-36.14 \%$ $(\mathrm{MSC} 3 \times \mathrm{JS} 5)$ to $6.49 \%(\mathrm{MSC} 2 \times \mathrm{AAUM} 3)$ over standard parent (Table 1). Four crosses showed significant positive better parent heterosis, 5 crosses showed significant positive mid parent heterosis whereas none of the crosses showed significant positive heterosis over standard parent. The highest significant positive better parent heterosis was observed in hybrid MSC1 $\times$ JS5 (20.22) followed by MSC3 $\times$ JS3 (19.83). The highest significant positive mid parent heterosis was observed in hybrid MSC3 $\times$ JS3 (29.26) followed by MSC1 × JS5 (28.24). Similar results were obtained by Kumar et al., (1989) for number of branches in marigold. Weerasekara et al., (2008) also showed similar percent of mid parent heterosis and heterobeltiosis for this trait in okra. Hassan et al., (2012) also reported highly positive significant average heterosis estimates in petunia. High results of heterosis for both primary and secondary branch number were observed by Panwar et al., (2013). Kumari et al., (2018) reported good amount of relative heterosis for number of branches per plant in China aster.

In ornamental crops like marigold, plant height parameter is an important determining factor for its utility as they are widely used in urban landscaping. Depending on their height, the tall plants with longer stalks can be used as a screening component and used as cut flowers, while the dwarf ones can be used as loose flower, landscaping, pot plants or edge plants. For plant height, the range of heterosis varied from $-22.90 \%$ (MSC2 $\times$ AAUM5) to $27.95 \%(\mathrm{MSC} 1 \times \mathrm{JS} 1)$ over better parent, $22.25 \%$ (MSC2 $\times$ AAUM5) to $36.89 \%$ (MSC1 $\times$ JS1) over mid parent and $-22.04 \%($ MSC2 $\times$ $\mathrm{PBG})$ to $17.78 \%(\mathrm{MSC} 3 \times$ AAUM4) over standard parent (Table 1). Out of 30 hybrids,
19 hybrids and 23 hybrids exhibited significant positive heterosis over better parent and mid parent, respectively. The $F_{1}$ hybrid MSC1 $\mathrm{x}$ JS1 showed the highest significant positive heterosis over mid parent and better parent with values $36.89 \%$ and $27.95 \%$, respectively. Sixteen hybrids exhibited significant and positive heterosis over standard parent, out of which, MSC3 x AAUM4 (17.78\%) showed highest significant positive heterosis over standard parent. The results were also in accordance with Pant and Lal (1992) in gladiolus, Hassan et al (2012) in petunia. According to Panwar et al., (2013) in marigold, 10 hybrids, 17 hybrids and 1 hybrid showed significant and positive heterobeltiosis, average and standard heterosis respectively. Bhargav et al., (2018) observed 20 crosses with significant positive relative heterosis and two crosses with significant negative relative heterosis among 30 crosses in China aster.

Marigolds are also being used for the bedding purpose; as such plant spread is one of the important factors which decide the density and planting distance. Less the plant spread, more is the density of plants in the given area. However, a plant with more plant spread is expected to produce more flowers per plant. Thus, both positive and negative heterosis can be considered depending upon the purpose. The range of heterosis for plant spread varied from $-21.33 \%$ (MSC1 $\times$ AAUM5) to $19.34 \%$ $(\mathrm{MSC} 2 \times \mathrm{JS} 3)$ over mid parent, $-24.33 \%$ $(\mathrm{MSC} 2 \times$ AAUM5) to $7.54 \%(\mathrm{MSC} 2 \times \mathrm{JS} 3)$ over better parent and $-18.56 \%(\mathrm{MSC} 3 \times \mathrm{JS} 3)$ to $7.98 \%($ MSC1 $\times$ AAUM3) (Table 2). Out of 30 hybrids, only three hybrids were recorded to have significant positive heterosis for plant spread over the mid parent and none of the hybrids showed significant positive heterosis over the better parent and standard parent. The negative heterosis for plant spread in hybrids is also a desirable trait. Eight hybrids, 17 hybrids and 12 hybrids showed 
significant and negative mid parent, better parent and standard heterosis for this trait respectively. Velmurugan (2003) reported three and four hybrids to have significant positive heterosis over mid parent and better parent respectively, while none of the hybrids showed significant positive heterosis over standard parent in marigold. Panwar et al., (2013) in marigold observed that the range of heterosis for plant spread varied from 40.52\% (MS $7 \times$ Af.Sel.1) to $8.77 \%$ (MS $5 \times$ Pusa Narangi Gainda) over better parent, $33.94 \%$ (MS $7 \times$ Af.Sel.1) to $16.72 \%$ (MS 5 $\times$ Pusa Narangi Gainda) over mid parent and $44.29 \%$ (MS $7 \times$ Af.Sel.1) to $9.93 \%$ (MS $8 \times$ Pusa Narangi Gainda) over commercial check.

Heterosis for early flowering is important in hybrids of flower crops and can largely enhance the economic yield when price differentials for early marketing are high for loose flowers and bedding materials (Singh and Misra, 2017). It increases the number of harvest of flowers. Hence, negative heterosis for days to $50 \%$ flowering is desirable. Heterosis for days to $50 \%$ flowering varied from $-16.01 \%(\mathrm{MSC} 1 \times \mathrm{JS} 2)$ to $23.84 \%$ $(\mathrm{MSC} 2 \times \mathrm{JS} 3),-22.87 \%(\mathrm{MSC} 1 \times \mathrm{JS} 2)$ to $19.98 \%($ MSC2 $\times$ AAUM5) and $-15.00 \%$ $(\mathrm{MSC} 1 \times \mathrm{JS} 2)$ to $31.35 \%(\mathrm{MSC} 2 \times \mathrm{JS} 5)$ over mid parent, better parent and standard parent, respectively (Table 2). Out of 30 hybrids, 5 hybrids displayed significant negative heterosis over mid and better parent, whereas 2 crosses exhibited significantly negative standard heterosis. The hybrid MSC1 x JS2 showed highest significant and desirable mid parent, better parent and standard parent heterosis for days to $50 \%$ flowering. Kumari et al., (2018) and Bhargav et al., (2018) have also reported earliness in China aster hybrids.

Duration of flowering is one of the important characters in any crop as it has the potential of producing more yields. Higher the flowering duration, more will be the production of flowers. Hence, positive heterosis for duration of flowering is of utmost importance. The range of mid parent, better parent and standard parent heterosis was $-10.01 \%$ $(\mathrm{MSC} 1 \times \mathrm{AAUM} 3)$ to $15.03 \%(\mathrm{MSC} 2 \times \mathrm{JS} 3)$, $-15.46 \%$ (MSC3 $\times$ AAUM4) to $9.45 \%$ $(\mathrm{MSC} 2 \times \mathrm{JS} 3)$ and $-6.72 \%$ to $11.67 \%$ (MSC3 $\times$ PNG), respectively for flowering duration. About 3 hybrids, 2 hybrids and 8 hybrids were observed to have significant positive average heterosis, heterobeltiosis and standard heterosis, respectively for flowering duration (Table 3).

The number of flowers per plant directly influences the yield of the crop plants; hence emphasis should be laid to develop hybrids that produce large number of flowers per plant. Thus, positive heterosis for flower size is of prime importance. For number of flowers per plant, the heterosis ranged from $31.13 \%(\mathrm{MSC} 2 \times \mathrm{JS} 2)$ to $38.31 \%(\mathrm{MSC} 3 \times$ $\mathrm{PBG}),-35.52 \%(\mathrm{MSC} 3 \times \mathrm{AAUM} 5)$ to $12.71 \%(\mathrm{MSC} 3 \times \mathrm{PNG})$ and $-37.44 \%$ (MSC3 $\times$ AAUM5) to $9.35 \%($ MSC3 $\times$ PNG) over mid parent, better parent and standard parent, respectively (Table 3 ). . About 10 hybrids, 3 hybrids and 3 hybrids were observed to have significant positive average heterosis, heterobeltiosis and standard heterosis, respectively for this trait. The hybrid MSC3 $\mathrm{x}$ PBG was found to have the highest value of heterosis over the mid parent. The hybrid MSC3 x PNG showed high heterosis over mid parent, better parent and standard parent. From plant breeder's point of view, economic heterosis is more relevant. It was observed that the hybrid MSC3 x PNG had the highest heterosis for number of flowers per plant and flower duration over the standard parent. Bhargav et al., (2018) reported that relative heterosis ranged from -60.88 (L5 x T2) to 108.79 (L6 x T5) and 6 crosses showed significant positive relative heterosis in China aster (Table 3). 
Table.1 Extent of heterosis for number of branches per plant and plant height

\begin{tabular}{|c|c|c|c|c|c|c|}
\hline \multirow[t]{2}{*}{ Genotypes } & \multicolumn{3}{|c|}{ Number of branches per plant } & \multicolumn{3}{|c|}{ Plant height } \\
\hline & MPH & BPH & SH & MPH & ВPH & SH \\
\hline MSC1 x PNG & $16.55 *$ & $16.03 *$ & 0.69 & $15.04 * *$ & $7.89 * *$ & 1.24 \\
\hline MSC1 $\times$ PBG & -12.22 & -12.69 & $-24.09 * *$ & $22.62 * *$ & $18.31 * *$ & 4.57 \\
\hline MSC1 $x$ AAUM3 & -4.26 & -5.55 & $-16.50 *$ & $-5.60 *$ & $-14.34 * *$ & $-13.61 * *$ \\
\hline MSC1 x AAUM4 & 4.69 & 3.05 & -11.37 & $22.69 * *$ & $10.24 * *$ & $13.66 * *$ \\
\hline MSC1 x AAUM5 & 8.88 & 5.13 & -2.88 & $20.45 * *$ & $8.90 * *$ & $10.73 * *$ \\
\hline MSC1 x JS1 & -4.3 & -10.53 & $-23.05 * *$ & $36.89 * *$ & $27.95 * *$ & 5.14 \\
\hline MSC1 x JS2 & -6.38 & -13.95 & $-25.99 * *$ & $18.86 * *$ & $7.25 * *$ & $9.53 * *$ \\
\hline MSC1 x JS3 & $25.10 * *$ & 15.44 & -0.71 & $12.08 * *$ & 1.76 & 2.48 \\
\hline MSC1 x JS4 & -6.82 & $-18.94 *$ & $-30.28 * *$ & $19.43 * *$ & $5.93 *$ & $12.46 * *$ \\
\hline MSC1 x JS5 & $28.24 * *$ & $20.22 *$ & 3.4 & $19.64 * *$ & $5.48 *$ & $13.57 * *$ \\
\hline MSC2 x PNG & 5.79 & -1.21 & -1.21 & 0.66 & -2.44 & -2.44 \\
\hline MSC2 $x$ PBG & $-19.74 * *$ & $-24.98 * *$ & $-24.98 * *$ & $-17.23 * *$ & $-22.04 * *$ & $-22.04 * *$ \\
\hline MSC2 $x$ AAUM3 & $13.04 *$ & 6.49 & 6.49 & $8.68 * *$ & $8.22 * *$ & $9.14 * *$ \\
\hline MSC2 x AAUM4 & 10.91 & 1.66 & 1.66 & $7.86 * *$ & $6.24 *$ & $9.53 * *$ \\
\hline MSC2 $\times$ AAUM5 & -1.3 & -5.06 & -5.06 & $-22.25 * *$ & $-22.90 * *$ & $-21.60 * *$ \\
\hline MSC2 x JS1 & $-20.03 * *$ & $-30.10 * *$ & $-30.10 * *$ & $31.20 * *$ & $12.46 * *$ & $12.46 * *$ \\
\hline MSC2 $\times$ JS2 & -6.92 & $-19.90 * *$ & $-19.90 * *$ & $12.15 * *$ & $10.99 * *$ & $13.35 * *$ \\
\hline MSC2 $\times$ JS3 & 12.26 & -3.05 & -3.05 & $11.14 * *$ & $10.74 * *$ & $11.53 * *$ \\
\hline MSC2 x JS4 & -8.7 & $-25.30 * *$ & $-25.30 * *$ & 0.71 & -2.21 & 3.81 \\
\hline MSC2 x JS5 & -9.36 & $-20.57 * *$ & $-20.57 * *$ & $6.21 * *$ & 2.43 & $10.29 * *$ \\
\hline MSC3 $x$ PNG & $19.40 * *$ & $18.29 *$ & 2.64 & $21.66 * *$ & $16.68 * *$ & $9.49 * *$ \\
\hline MSC3 x PBG & $-21.90 * *$ & $-22.70 * *$ & $-32.79 * *$ & $9.20 * *$ & $7.83 *$ & -4.7 \\
\hline MSC3 x AAUM3 & 1.04 & -0.82 & -12.31 & $9.79 * *$ & 1.8 & 2.66 \\
\hline MSC3 x AAUM4 & -0.01 & -1.09 & $-15.77 *$ & $24.46 * *$ & $14.24 * *$ & $17.78 * *$ \\
\hline MSC3 x AAUM5 & -10.75 & -14.24 & $-20.78 * *$ & $22.00 * *$ & $12.69 * *$ & $14.59 * *$ \\
\hline MSC3 x JS1 & 7.21 & 0.7 & $-14.25 *$ & $32.81 * *$ & $21.46 * *$ & 4.66 \\
\hline MSC3 x JS2 & 0.8 & -6.92 & $-20.74 * *$ & $-8.38 * *$ & $-15.54 * *$ & $-13.75 * *$ \\
\hline MSC3 $x$ JS3 & $29.26 * *$ & $19.83 *$ & 2.05 & $-6.88 * *$ & $-13.61 * *$ & $-12.99 * *$ \\
\hline MSC3 x JS4 & -10.69 & $-21.97 * *$ & $-33.56 * *$ & $16.49 * *$ & $5.51 *$ & $12.02 * *$ \\
\hline MSC3 x JS5 & $-20.38 * *$ & $-25.01 * *$ & $-36.14 * *$ & $16.86 * *$ & $5.19 *$ & $13.26 * *$ \\
\hline SEd & 1.04 & 1.20 & 1.20 & 1.72 & 1.99 & 1.99 \\
\hline
\end{tabular}

(MPH= Mid Parent Heterosis, BPH= Better Parent Heterosis, $\mathrm{SH}=$ Standard Heterosis)

* Significant at $5 \%$ level, $* *$ Significant at $1 \%$ level 
Table.2 Extent of heterosis for Days to 50\% flowering and Plant spread

\begin{tabular}{|c|c|c|c|c|c|c|}
\hline \multirow[t]{2}{*}{ Genotypes } & \multicolumn{3}{|c|}{ Days to $50 \%$ flowering } & \multicolumn{3}{|c|}{ Plant spread } \\
\hline & MPH & BPH & SH & MPH & BPH & SH \\
\hline MSC1 $\times$ PNG & 3.34 & 0.79 & $16.84 * *$ & 2.5 & -2.03 & 5.54 \\
\hline MSC1 x PBG & 0.97 & -0.09 & $12.47 * *$ & -5.42 & $-9.15 *$ & -2.13 \\
\hline MSC1 x AAUM3 & -1.45 & -4.2 & 5.59 & 0.81 & 0.23 & 7.98 \\
\hline MSC1 x AAUM4 & $19.74 * *$ & $15.84 * *$ & $27.67 * *$ & -4.7 & $-10.35 *$ & -3.41 \\
\hline MSC1 x AAUM5 & -0.45 & -4.81 & 4.91 & $-21.33 * *$ & $-21.73 * *$ & $-14.82 * *$ \\
\hline MSC1 x JS1 & $-8.09 * *$ & $-13.13 * *$ & -4.26 & $-12.05 * *$ & $-20.57 * *$ & $-14.43 * *$ \\
\hline MSC1 x JS2 & $-16.01 * *$ & $-22.87 * *$ & $-15.00 * *$ & 3.68 & $-8.97 *$ & -1.93 \\
\hline MSC1 x JS3 & $-8.86 * *$ & $-9.38 * *$ & -0.13 & 5.38 & $-8.07 *$ & -0.97 \\
\hline MSC1 x JS4 & $-7.97 * *$ & $-10.95 * *$ & -1.85 & 5.16 & $-10.94 * *$ & -4.06 \\
\hline MSC1 x JS5 & 0.6 & -2.88 & $15.00 * *$ & $-12.86 * *$ & $-22.79 * *$ & $-16.82 * *$ \\
\hline MSC2 $\times$ PNG & -4.04 & $-10.63 * *$ & 3.6 & -1.37 & -2.26 & -2.26 \\
\hline MSC2 $\times$ PBG & $5.34 *$ & -0.54 & $11.96 * *$ & -1.62 & -2 & -2 \\
\hline MSC2 x AAUM3 & $15.25 * *$ & $13.00 * *$ & $17.60 * *$ & -1.47 & -4.48 & 1.74 \\
\hline MSC2 x AAUM4 & $18.19^{* *}$ & $16.45 * *$ & $19.98 * *$ & $-11.10 * *$ & $-13.34 * *$ & $-13.34 * *$ \\
\hline MSC2 $\times$ AAUM5 & $20.31 * *$ & $19.98 * *$ & $20.64 * *$ & $-21.14 * *$ & $-24.33 * *$ & $-17.65 * *$ \\
\hline MSC2 x JS1 & 2.41 & 1.45 & 1.45 & -4.34 & $-10.63 *$ & $-10.63 *$ \\
\hline MSC2 x JS2 & 4.03 & -0.03 & -0.03 & -2.06 & $-11.15 *$ & $-11.15 *$ \\
\hline MSC2 $\times$ JS3 & $23.84 * *$ & $18.75 * *$ & $29.38 * *$ & $19.34 * *$ & 7.54 & 7.54 \\
\hline MSC2 x JS4 & $9.29 * *$ & $7.65 *$ & $10.97 * *$ & 4.5 & $-8.70 *$ & $-8.70 *$ \\
\hline MSC2 x JS5 & $20.28 * *$ & $10.93 * *$ & $31.35 * *$ & 4.68 & -4.12 & -4.12 \\
\hline MSC3 $\times$ PNG & $7.01 * *$ & -1.05 & $14.70 * *$ & -0.73 & -3.59 & 0.45 \\
\hline MSC3 $\times$ PBG & $11.90 * *$ & 4.89 & $18.07 * *$ & -2.82 & -5.13 & -1.16 \\
\hline MSC3 $\times$ AAUM3 & 2.34 & -0.42 & 3.63 & -5.02 & -6.05 & 0.06 \\
\hline MSC3 $\times$ AAUM4 & $10.49 * *$ & $8.04 *$ & $11.32 * *$ & $-12.07 * *$ & $-15.96 * *$ & $-12.44 * *$ \\
\hline MSC3 $\times$ AAUM5 & $16.19 * *$ & $14.98 * *$ & $15.62 * *$ & -3.51 & -5.57 & 2.77 \\
\hline MSC3 x JS1 & 2.44 & 2.27 & 0.69 & $-10.62 * *$ & $-18.06 * *$ & $-14.63 * *$ \\
\hline MSC3 x JS2 & 4.56 & 1.24 & -0.32 & $12.25 * *$ & 0 & 4.19 \\
\hline MSC $3 \times$ JS3 & $16.16 * *$ & $10.56 * *$ & $20.46 * *$ & $-11.67 * *$ & $-21.83 * *$ & $-18.56 * *$ \\
\hline MSC3 x JS4 & $15.92 * *$ & $13.32 * *$ & $16.81 * *$ & $14.87 * *$ & -1.36 & 2.77 \\
\hline MSC3 x JS5 & $9.30 * *$ & 0.09 & $18.51 * *$ & -4.13 & $-13.79 * *$ & $-10.18 *$ \\
\hline SEd & 1.56 & 1.81 & 1.81 & 1.92 & 2.22 & 2.22 \\
\hline
\end{tabular}

(MPH= Mid Parent Heterosis, BPH= Better Parent Heterosis, $\mathrm{SH}=$ Standard Heterosis)

* Significant at $5 \%$ level, ** Significant at $1 \%$ level 
Table.3 Extent of heterosis for duration of flowering and number of flowers per plant

\begin{tabular}{|c|c|c|c|c|c|c|}
\hline \multirow[t]{2}{*}{ Genotypes } & \multicolumn{3}{|c|}{ Duration of flowering } & \multicolumn{3}{|c|}{ Number of flowers per plant } \\
\hline & MPH & BPH & SH & МРH & BPH & SH \\
\hline MSC1 x PNG & -3.17 & $-6.81 *$ & $9.38 *$ & $28.93 * *$ & 6.99 & 4.55 \\
\hline MSC1 $\times$ PBG & -5.94 & $-11.40 * *$ & 3.99 & $23.51 * *$ & -0.47 & -2.73 \\
\hline MSC1 $x$ AAUM3 & $-10.01 * *$ & $-14.05 * *$ & 0.89 & $12.11 * *$ & $9.95 *$ & 7.45 \\
\hline MSC1 $\times$ AAUM4 & -1.87 & $-12.16 * *$ & 3.11 & -8.36 & $-24.26 * *$ & $-25.98 * *$ \\
\hline MSC1 x AAUM5 & -4.99 & $-13.67 * *$ & 1.33 & $30.08 * *$ & 3.26 & 0.91 \\
\hline MSC1 x JS1 & -4.72 & $-8.43 *$ & 7.48 & $-16.37 * *$ & $-22.69 * *$ & $-24.45 * *$ \\
\hline MSC1 x JS2 & -3.43 & $-10.16 * *$ & 5.45 & $-17.61 * *$ & $-21.89 * *$ & $-14.81 * *$ \\
\hline MSC1 x JS3 & 1.92 & $-9.83 * *$ & 5.83 & $13.80 * *$ & $9.65 *$ & 7.16 \\
\hline MSC1 x JS4 & -4.14 & $-9.89 * *$ & 5.77 & -0.2 & $-15.92 * *$ & $-17.83 * *$ \\
\hline MSC1 x JS5 & -4.05 & $-7.13 *$ & $9.00 *$ & -5.55 & $-8.79 *$ & -4.3 \\
\hline MSC2 $\times$ PNG & 2.4 & -1.64 & 6.79 & $21.21 * *$ & -0.33 & -0.33 \\
\hline MSC2 x PBG & 2.89 & 1.04 & 4.82 & $23.15 * *$ & -1.61 & -1.61 \\
\hline MSC2 $\times$ AAUM3 & 4.9 & 1.54 & $8.50 *$ & 2.09 & -0.99 & -0.99 \\
\hline MSC2 $\times$ AAUM4 & $10.66 * *$ & 6.66 & 6.66 & $-15.60 * *$ & $-30.86 * *$ & $-30.86 * *$ \\
\hline MSC2 x AAUM5 & $9.51 * *$ & 7.29 & 7.29 & -4.23 & $-24.62 * *$ & $-24.62 * *$ \\
\hline MSC2 x JS1 & 3.59 & -0.35 & $7.86 *$ & -6.56 & $-14.52 * *$ & $-14.52 * *$ \\
\hline MSC2 x JS2 & 2.59 & 2.07 & 3.11 & $-31.13 * *$ & $-33.99 * *$ & $-28.01 * *$ \\
\hline MSC2 x JS3 & $15.03 * *$ & $9.45 *$ & $9.45 *$ & $-12.61 * *$ & $-16.71 * *$ & $-16.71 * *$ \\
\hline x JS4 & 1.56 & -0.06 & 3.23 & $17.67 * *$ & -1.78 & -1.78 \\
\hline MSC2 x JS5 & 2.45 & -2.14 & 7.48 & $-13.26 * *$ & $-15.30 * *$ & $-11.13 *$ \\
\hline MSC3 $\times$ F & 2.03 & 1.21 & $11.67 * *$ & $35.43 * *$ & $12.71 * *$ & $9.35 *$ \\
\hline MSC3 x PBG & -3.55 & -6.44 & 3.23 & $38.31 * *$ & $11.77 *$ & 8.44 \\
\hline $\operatorname{MSC} 3 \times \mathbf{A}$ & -1.02 & -2.59 & 7.48 & 2.43 & 0.81 & -2.19 \\
\hline MSC3 $\times$ AAUM4 & $-8.15 *$ & $-15.46 * *$ & -6.72 & $-10.12 *$ & $-25.50 * *$ & $-27.72 * *$ \\
\hline MSC3 $\times$ AAUM5 & -1.81 & $-8.22 *$ & 1.27 & $-18.99 * *$ & $-35.52 * *$ & $-37.44 * *$ \\
\hline MSC3 x JS1 & 2.18 & 1.21 & $11.67 * *$ & $-17.20 * *$ & $-23.20 * *$ & $-25.49 * *$ \\
\hline MSC3 x JS2 & -6.15 & $-10.11 * *$ & -0.82 & $-22.75 * *$ & $-27.01 * *$ & $-20.40 * *$ \\
\hline MSC3 x JS3 & 5.94 & -3.68 & 6.28 & $-13.61 * *$ & $-16.46 * *$ & $-18.95 * *$ \\
\hline MSC3 x JS4 & 1.28 & -1.95 & $8.18 *$ & -6.23 & $-20.77 * *$ & $-23.13 * *$ \\
\hline MSC3 x JS5 & -3.12 & -3.34 & 6.65 & -4.86 & -8.44 & -3.93 \\
\hline SEd & 1.78 & 2.05 & 2.05 & 3.18 & 3.67 & 3.67 \\
\hline
\end{tabular}

(MPH= Mid Parent Heterosis, BPH= Better Parent Heterosis, $\mathrm{SH}=$ Standard Heterosis)

* Significant at $5 \%$ level, ** Significant at $1 \%$ level 
Table.4 Extent of heterosis for flower weight and flower size

\begin{tabular}{|c|c|c|c|c|c|c|}
\hline \multirow[t]{2}{*}{ Genotypes } & \multicolumn{3}{|c|}{ Flower weight } & \multicolumn{3}{|c|}{ Flower size } \\
\hline & МPH & BPH & SH & MPH & ВPH & SH \\
\hline MSC1 1 PNG & $9.47 * *$ & 4.21 & $6.85 * *$ & $3.18 * *$ & $2.70 *$ & $9.83 * *$ \\
\hline MSC1 $\times$ PBG & $13.34 * *$ & $9.15 * *$ & $11.91 * *$ & 1.87 & 0.21 & $7.17 * *$ \\
\hline MSC1 1 AAUM3 & -0.02 & -0.17 & 2.66 & $-11.66 * *$ & $-12.00 * *$ & $-5.89 * *$ \\
\hline MSC1 $\times$ AAUM4 & $14.27 * *$ & $10.17 * *$ & $12.95 * *$ & $17.96 * *$ & $14.81 * *$ & $22.78 * *$ \\
\hline MSC1 x AAUM5 & $11.25 * *$ & $8.93 * *$ & $11.69 * *$ & $16.75 * *$ & $12.78 * *$ & $20.61 * *$ \\
\hline MSC1 x JS1 & $18.21 * *$ & $-27.95 * *$ & $-26.12 * *$ & $-13.28 * *$ & $-28.62 * *$ & $-23.67 * *$ \\
\hline MSC1 1 JS2 & $-28.60 * *$ & $-51.64 * *$ & $-50.41 * *$ & $18.88 * *$ & $-8.10 * *$ & -1.72 \\
\hline MSC1 1 JS3 & $8.96 * *$ & $-12.59 * *$ & $-10.38 * *$ & $21.62 * *$ & $-10.13 * *$ & $-3.89 * *$ \\
\hline MSC1 x JS4 & 1.85 & $-25.18 * *$ & $-23.29 * *$ & $-22.89 * *$ & $-29.92 * *$ & $-25.06 * *$ \\
\hline MSC1 x JS5 & -5.25 & $-35.47 * *$ & $-33.84 * *$ & $16.32 * *$ & $-4.83 * *$ & 1.78 \\
\hline MSC2 $\times$ PNG & $18.24 * *$ & $13.91 * *$ & $13.91 * *$ & $9.52 * *$ & $6.45 * *$ & $12.78 * *$ \\
\hline MSC2 $\times$ PBG & $17.00 * *$ & $14.04 * *$ & $14.04 * *$ & $12.02 * *$ & $10.15 * *$ & $13.94 * *$ \\
\hline MSC2 $\times$ AAUM3 & $18.04 * *$ & $16.41 * *$ & $19.71 * *$ & $13.26 * *$ & $10.00 * *$ & $16.72 * *$ \\
\hline MSC2 $\times$ AAUM4 & $25.81 * *$ & $22.76 * *$ & $22.76 * *$ & $20.87 * *$ & $20.14 * *$ & $21.61 * *$ \\
\hline MSC2 $\times$ AAUM5 & $24.37 * *$ & $23.29 * *$ & $23.29 * *$ & $18.64 * *$ & $18.44 * *$ & $18.44 * *$ \\
\hline MSC2 x JS1 & $39.89 * *$ & $-14.35 * *$ & $-14.35 * *$ & $-11.17 * *$ & $-24.89 * *$ & $-24.89 * *$ \\
\hline MSC2 $\times$ JS2 & $10.71 * *$ & $-24.51 * *$ & $-24.51 * *$ & $9.51 * *$ & $-13.28 * *$ & $-13.28 * *$ \\
\hline MSC2 $\times$ JS3 & $19.33 * *$ & -3.36 & -3.36 & $22.35 * *$ & $-7.56 * *$ & $-7.56 * *$ \\
\hline MSC2 x JS4 & $13.43 * *$ & $-16.01 * *$ & $-16.01 * *$ & $-5.39 * *$ & $-11.33 * *$ & $-11.33 * *$ \\
\hline MSC2 x JS5 & $-10.56 * *$ & $-38.68 * *$ & $-38.68 * *$ & $12.26 * *$ & $-5.67 * *$ & $-5.67 * *$ \\
\hline MSC3 $\times$ PNG & $16.38 * *$ & $11.19 * *$ & $13.13 * *$ & $19.59 * *$ & $15.55 * *$ & $31.28 * *$ \\
\hline MSC3 $x$ PBG & -1.64 & $-4.93 *$ & -3.27 & $13.39 * *$ & $8.31 * *$ & $23.06 * *$ \\
\hline MSC3 x AAUM3 & $-4.28 *$ & $-4.79 *$ & -2.09 & $13.93 * *$ & $10.17 * *$ & $25.17 * *$ \\
\hline MSC3 $\times$ AAUM4 & $16.77 * *$ & $12.99 * *$ & $14.96 * *$ & $23.51 * *$ & $16.77 * *$ & $32.67 * *$ \\
\hline MSC3 x AAUM5 & $22.29 * *$ & $20.19 * *$ & $22.29 * *$ & $18.36 * *$ & $11.10 * *$ & $26.22 * *$ \\
\hline MSC3 x JS1 & $15.66 * *$ & $-29.40 * *$ & $-28.17 * *$ & -1.61 & $-20.88 * *$ & $-10.11 * *$ \\
\hline MSC3 $x$ JS2 & $47.08 * *$ & -0.17 & 1.57 & $29.20 * *$ & $-2.20 *$ & $11.11 * *$ \\
\hline MSC3 $x$ JS3 & $7.25 * *$ & $-13.72 * *$ & $-12.21 * *$ & $24.52 * *$ & $-9.73 * *$ & $2.56 *$ \\
\hline MSC3 $\times$ JS4 & $-11.41 * *$ & $-34.76 * *$ & $-33.62 * *$ & $-10.42 * *$ & $-20.73 * *$ & $-9.94 * *$ \\
\hline MSC3 x JS5 & -2.51 & $-33.48 * *$ & $-32.32 * *$ & $13.46 * *$ & $-9.29 * *$ & $3.06 *$ \\
\hline SEd & 0.16 & 0.18 & 0.18 & 0.063 & 0.073 & 0.073 \\
\hline
\end{tabular}

(MPH= Mid Parent Heterosis, BPH= Better Parent Heterosis, SH=Standard Heterosis)

* Significant at $5 \%$ level, ** Significant at $1 \%$ level 
Table.5 Extent of heterosis for flower yield per plant and vase life

\begin{tabular}{|c|c|c|c|c|c|c|}
\hline \multirow[t]{2}{*}{ Genotypes } & \multicolumn{3}{|c|}{ Flower yield per plant } & \multicolumn{3}{|l|}{ Vase life } \\
\hline & MPH & ВPH & SH & МPH & ВPH & SH \\
\hline MSC1 $\times$ PNG & $36.85 * *$ & $20.72 * *$ & $5.13 * *$ & $8.91 *$ & 1.85 & -4.07 \\
\hline MSC1 x PBG & $43.52 * *$ & $22.05 * *$ & $6.29 * *$ & $12.54 * *$ & 2.47 & -3.49 \\
\hline MSC1 $\times$ AAUM3 & $11.64 * *$ & 1.51 & $8.01 * *$ & 2.8 & 1.85 & -4.07 \\
\hline MSC1 $\times$ AAUM4 & $-8.48 * *$ & $-17.47 * *$ & $-28.12 * *$ & $26.53 * *$ & $14.81 * *$ & 8.14 \\
\hline MSC1 x AAUM5 & $42.93 * *$ & $24.36 * *$ & $8.30 * *$ & $29.49 * *$ & $17.90 * *$ & $11.05 *$ \\
\hline MSC1 x JS1 & $-25.88 * *$ & $-44.08 * *$ & $-51.30 * *$ & $-23.42 * *$ & $-36.42 * *$ & $-40.12 * *$ \\
\hline MSC1 x JS2 & $-28.64 * *$ & $-43.69 * *$ & $-50.96 * *$ & $-24.54 * *$ & $-36.42 * *$ & $-40.12 * *$ \\
\hline MSC1 1 JS3 & $-10.41 * *$ & $-28.18 * *$ & $-37.45 * *$ & -4.18 & $-22.22 * *$ & $-26.74 * *$ \\
\hline MSC1 1 JS4 & $-21.83 * *$ & $-39.83 * *$ & $-47.60 * *$ & $-22.60 * *$ & $-22.84 * *$ & $-27.33 * *$ \\
\hline MSC1 x JS5 & $-9.15 * *$ & $-27.60 * *$ & $-36.95 * *$ & $-12.66 * *$ & $-14.81 * *$ & $-19.77 * *$ \\
\hline MSC2 x PNG & $26.47 * *$ & $5.32 * *$ & $5.32 * *$ & 2.88 & -6.4 & -6.4 \\
\hline MSC2 x PBG & $30.56 * *$ & $5.12 * *$ & $5.12 * *$ & 5.57 & -6.4 & -6.4 \\
\hline MSC2 $\times$ AAUM3 & 0.4 & -2.62 & $3.61 *$ & $12.99 * *$ & $8.72 *$ & $8.72 *$ \\
\hline MSC2 x AAUM4 & $-9.24 * *$ & $-22.86 * *$ & $-22.86 * *$ & $26.97 * *$ & $12.21 * *$ & $12.21 * *$ \\
\hline MSC2 x AAUM5 & $6.49 * *$ & $-12.43 * *$ & $-12.43 * *$ & $15.41 * *$ & 2.33 & 2.33 \\
\hline MSC2 x JS1 & $-20.61 * *$ & $-42.71 * *$ & $-42.71 * *$ & -8.24 & $-25.58 * *$ & $-25.58 * *$ \\
\hline MSC2 $\times$ JS2 & $-14.14 * *$ & $-35.45 * *$ & $-35.45 * *$ & 7.42 & $-11.63 * *$ & $-11.63 * *$ \\
\hline MSC2 $\times$ JS3 & $11.28 * *$ & $-15.13 * *$ & $-15.13 * *$ & $13.55 * *$ & $-9.88 *$ & $-9.88 *$ \\
\hline MSC2 x JS4 & $-10.85 * *$ & $-34.48 * *$ & $-34.48 * *$ & $-14.71 * *$ & $-17.44 * *$ & $-17.44 * *$ \\
\hline MSC2 x JS5 & $15.77 * *$ & $-12.18 * *$ & $-12.18 * *$ & $-18.40 * *$ & $-22.67 * *$ & $-22.67 * *$ \\
\hline MSC3 $\times$ PNG & $31.16 * *$ & $10.96 * *$ & $6.72 * *$ & $22.29 * *$ & $10.98 *$ & $11.63 * *$ \\
\hline MSC3 x PBG & $31.84 * *$ & $7.75 * *$ & $3.63 *$ & $26.14 * *$ & $11.56 * *$ & $12.21 * *$ \\
\hline MSC3 x AAUM3 & $5.46 * *$ & 0.4 & $6.82 * *$ & $31.93 * *$ & $26.59 * *$ & $27.33 * *$ \\
\hline MSC3 x AAUM4 & $-5.01 * *$ & $-17.95 * *$ & $-21.09 * *$ & $20.00 * *$ & 5.78 & 6.4 \\
\hline MSC3 x AAUM5 & $14.63 * *$ & $-4.27 *$ & $-7.93 * *$ & $38.56 * *$ & $22.54 * *$ & $23.26 * *$ \\
\hline MSC3 x JS1 & $-25.15 * *$ & $-45.33 * *$ & $-47.42 * *$ & $-25.00 * *$ & $-39.31 * *$ & $-38.95 * *$ \\
\hline MSC3 x JS2 & $-15.80 * *$ & $-35.86 * *$ & $-38.31 * *$ & $-28.17 * *$ & $-41.04 * *$ & $-40.70 * *$ \\
\hline MSC3 $\times$ JS3 & $-9.05 * *$ & $-29.68 * *$ & $-32.37 * *$ & $12.41 * *$ & $-10.98 *$ & $-10.47 *$ \\
\hline MSC3 x JS4 & $-8.41 * *$ & $-31.84 * *$ & $-34.45 * *$ & $14.37 * *$ & $10.40 *$ & $11.05 *$ \\
\hline MSC3 $\times$ JS5 & $-26.89 * *$ & $-43.79 * *$ & $-45.94 * *$ & 3.98 & -1.73 & -1.16 \\
\hline SEd & 7.63 & 8.81 & 8.81 & 0.21 & 0.24 & 0.24 \\
\hline
\end{tabular}

(MPH= Mid Parent Heterosis, BPH= Better Parent Heterosis, $\mathrm{SH}=$ Standard Heterosis)

* Significant at $5 \%$ level, ** significant at $1 \%$ level

From garland making or loose flower market point of view, the weight of the flower is the dominant factor as higher the flower weight, more the price and market value. Also, it can be considered that a flower with more weight is likely to contain more number of seeds. So, positive heterosis for flower weight in marigold is desirable. For flower weight, the heterosis ranged from $-28.60 \%(\mathrm{MSC} 1 \times \mathrm{JS} 2)$ to $47.08 \%(\mathrm{MSC} 3 \times \mathrm{JS} 2),-51.64 \%(\mathrm{MSC} 1 \times$ JS2) to $23.29 \%$ (MSC2 $\times$ AAUM5) and $50.41 \%(\mathrm{MSC} 1 \times \mathrm{JS} 2)$ to $23.29 \%(\mathrm{MSC} 2 \times$ 
AAUM5) over mid parent, better parent and standard parent, respectively (Table 4). Out of 30 hybrids, 21 hybrids showed significant and positive average heterosis, whereas 11 hybrids and 12 hybrids showed significant and positive heterobeltiosis and standard heterosis.

Increased flower size is a very important aspect from the vase life of flower point of view (Singh and Misra, 2017). From the aspect of marketing, flowers with greater diameter are more preferred by customers than the smaller ones. Maximum number of hybrids was recorded to have significantly positive heterosis for flower size. The heterosis value ranged from $-22.89 \%$ (MSC1× JS4) to $29.20 \%($ MSC3 $\times$ JS2), $-29.92 \%$ $(\mathrm{MSC} 1 \times \mathrm{JS} 4)$ to $20.14 \%(\mathrm{MSC} 2 \times$ AAUM4) and $-25.06 \%(\mathrm{MSC} 1 \times \mathrm{JS} 4)$ to $32.67 \%$ (MSC3× AAUM4) for mid parent, better parent and standard heterosis (Table 4). Out of 30 hybrids, 22 hybrids showed significant and positive average heterosis, whereas 13 hybrids and 14 hybrids showed significant and positive heterobeltiosis and standard heterosis. The hybrids MSC1 x AAUM5, MSC2 x AAUM3, MSC2 x AAUM4, MSC3 x PNG, MSC3 x PBG, MSC3 x AAUM3, and MSC3 $x$ AAUM5 showed high significant and positive heterosis for both flower size and vase life of flower. Similarly, the hybrids MSC1 x AAUM4, MSC2 x AAUM3, MSC2 x AAUM4, MSC2 x AAUM5, MSC2 x PNG, MSC2 x PBG, MSC2 x AAUM3, MSC3 x PNG, MSC3 $x$ AAUM4, and MSC3 $x$ AAUM5 had high significant and positive values of heterosis for flower weight (Table 4). This indicated the positive correlation of flower size, flower weight and vase life of flower. Similar results were also obtained by Singh and Misra (2017) in marigold. Singh and Swarup (1971) observed the maximum heterosis in total number of flowers. The crosses $\mathrm{P}_{1} \times \mathrm{P}_{5}, \mathrm{P}_{1} \times \mathrm{P}_{6}$ and $\mathrm{P}_{4} \times \mathrm{P}_{6}$ showed the highest heterosis percentage in flower size and flower weight, early flowering and total number of flowers respectively.

Higher yield is necessary to reduce the import of flowers to the state and improve the economic status of farmers for the overall benefit of the state and the country as well. For the trait flower yield per plant, heterosis ranged from $-28.64 \%$ (MSC1× JS2) to $43.52 \%(\mathrm{MSC} 1 \times \mathrm{PBG}),-45.33 \%(\mathrm{MSC} 3 \times$ JS1) to $24.36 \%(\mathrm{MSC} 1 \times$ AAUM5) and $51.30 \%(\mathrm{MSC} 1 \times \mathrm{JS} 1)$ to $8.30 \%(\mathrm{MSC} 1 \times$ AAUM5) over mid parent, better parent and standard parent (Table 5). Out of 30 hybrids, 13 hybrids showed significant positive average heterosis, whereas 7 hybrids and 10 hybrids showed significant and positive heterobeltiosis and standard heterosis respectively. The highest significantly positive heterosis over mid parent, better parent and standard parent was recorded in the hybrid, MSC1 x AAUM5, followed by the hybrids MSC1 x PBG and MSC1 x PNG which had highly significant positive heterosis (mid parent and better parent) for the trait. Similar findings were reported by Velmurugan (2003), Namita (2008), Panwar et al., (2013) in marigold. The maximum heterosis over better parent was $24.36 \%$ in the hybrid MSC1 x AAUM5 and 22.05\% in MSC1 1 PBG for the trait flower yield per plant. The parents MSC1, AAUM5 and PBG were geographically diverse; however, heterosis has frequently been related to the degree of genetic diversity of parents crossed (Timothy (1963), Vanderberg and Matzinger (1970) and Swarup et al., (1975).

The hybrids with high yield cannot alone fulfil the requirements of the producers as well as the consumers. Hence, quality of the produce is a prerequisite. Considering this point, the trait, vase life of flowers, is necessary in hybrid development programme. For vase life of flowers, heterosis ranged from $-28.17 \%$ (MSC3× JS2) to $38.56 \%$ (MSC3 $\times$ 
AAUM5), $-41.04 \%(\mathrm{MSC} 3 \times \mathrm{JS} 2)$ to $17.90 \%$ $(\mathrm{MSC} 1 \times$ AAUM5) and $-40.70 \%(\mathrm{MSC} 3 \times$ JS2) to $27.33 \%($ MSC3 $\times$ AAUM3) over mid parent, better parent and standard parent (Table 5). Out of 30 hybrids, 15 hybrids showed significant positive average heterosis, whereas 9 hybrids and 8 hybrids showed significant and positive heterobeltiosis and standard heterosis, respectively. MSC3 $\mathrm{x}$ AAUM5 and MSC3 x AAUM3 showed high heterosis values. These crosses can be exploited for developing high quality hybrids. Kulkarni et al., (2015) observed four best hybrids, out of twenty, to have high heterosis over mid parent and better parent for vase life in China aster. Out of 30 crosses made by Bhargav et al., (2018), four crosses showed significantly positive heterosis in China aster.

The $\mathrm{F}_{1}$ hybrids namely, MSC1 x AAUM5, MSC1 $x$ AAUM3, MSC3 $x$ AAUM3 and MSC3 x PNG showing significant standard parent heterosis for flower yield were probably due to the highly significant heterotic effect of individual yield and its contributing traits like number of flowers per plant, flower duration, flower size and flower weight.

Hence, it is concluded that heterosis can be exploited for growth and flowering traits in African marigold. As the traits used in the study were the essential traits which indirectly influenced the potentiality of the crop for enhanced production, therefore breeding activities can be initiated to develop $F_{1}$ hybrids with varied flowering related traits. The parents can be used in future breeding programme to obtain high heterotic expression for different traits and also to recover desirable transgressive segregants in the subsequent generations. The highly heterotic hybrids can be further selected based on the trait requirement for exploitation of heterosis.

\section{Acknowledgments}

The authors are thankful to the Head, Department of Plant Breeding and Genetics, Assam Agricultural University, Jorhat for providing the research facilities for field work. The authors are also thankful to the Directorate of Postgraduate Studies, Assam Agricultural University, Jorhat.

\section{References}

Bhargav, V.; Kumar, R.; Rao, T. M.; Bharathi, T. U. and Venugopalan, R. (2018). Estimation of Relative Heterosis in $\mathrm{F}_{1}$ Hybrids of China Aster [Callistephus chinensis (L.) Nees]. Int.J.Curr.Microbiol.App.Sci. 7(12): 1225-1232.

Hassan, B.; Hossein, N.; Abdolreza, B.; Ali, T. and Marjan, S. (2012). Estimation of heterosis and combining ability in petunia (Petunia hybrida Hort.). Notulae Scientia Biologicae 4(3): 1517.

Hays, H. K.; Immer, I. R. and Smith, O. C. (1955). Methods of plant breeding. McGraw Hill Company Inc., New York. 525-565.

Kempthorne, O. (1957). An introduction to genetic statistics. John Wiley and son, Inc. New York, pp. 73-98

Kulkarni, A. (2015). Heterosis and combining ability studies in China aster (Callistephus chinensis L. Nees). M.Sc thesis submitted to the University of Horticultural Sciences, Bagalkot.

Kumar, S.; Shanmgavelu, K. G. and Irulapan, I. (1989). Hybrid vigour in marigold for economic characters. S. Indian Hort., 38 (3): 173-174.

Kumari, P.; Kumar, R.; Rao, T. M.; Bharathi, T. U.; Dananjaya, M. V. and Bhargav, V. (2018). Exploitation of heterosis for growth, flower quality and yield traits in China aster (Callistephus chinensis). 
Indian Journal of Agricultural Sciences. 88(3): 453-457.

Namita. (2008). Interspecific hybridization in marigold. Ph.D Thesis submitted to Indian Agricultural Research Institute, New Delhi-12.

Pant, C. C. and Lal, S. D. (1992). Heterosis in gladiolus (Gladiolus primulinus Baker). Progressive Horticulture 24(3-4): 124 7.

Panwar, S.; Singh, K. P.; Janakjram, T. and Bharadwaj, C. (2013). Exploitation of heterosis for growth related traits in African marigold (Tagetes erecta). Indian Journal of Agricultural Sciences, 83(7), 728-733.

Phillips, R. L. (1999). Research needs in heterosis. In: J. G. and S. Pandey (eds.), Genetics and Exploitation of Heterosis in Crops, pp: 501-508.

Singh, D. and Misra, K. K. (2017). Heterosis breeding among different crosses in marigold (Tagetes spp.). Contemporary Research in India. 7(1).

Singh, B. and Swarup, V. (1971). Heterosis and combining ability in African marigold. Indian J. Genet. Plant Breed., 31 (9): 407-415.

Swarup, V.; Raghava, S. P. S. and
Balakrishnan, K. A. (1975). Heterosis in balsam. Indian Journal of Genetics and Plant Breeding, 35(1): 69-75.

Timothy, D. H. (1963). Genetic diversity, heterosis and use of exotic stocks of maize in Columbia. In: Statistical Genetics and Plant Breeding, NACNRC Publn. No. 982, pp.581-593.

Turner, J. R. (1953). A study on heterosis in upland cotton; II. Combining ability and inbreeding effects. Agron. J., 45:487490.

Vanderberg, P. and Matzinger, D. F. (1970). Genetic and heterosis in Nicotiana III. Crosses among introductions and fluecured varieties. Crop Sci., 10: 437-440.

Velmurugan, K.; Vijayakumar, M. and Jawaharlal, M. (2003). Heterosis and combining ability effects for xanthophyll content of marigold (Tagetes erecta L.). S. Indian Hort., 51(1-6): 237-240.

Weerasekara, D.; Jagadeesha, R. C.; Wali, M. C.; Salimath, P. M.; Hosamani, R. M. and Kalappanawar, I. K. (2008). Heterosis for yield and yield components in okra. Karnataka Journal of Agricultural Sciences 21(4): 578-9.

\section{How to cite this article:}

Chayanika Lahkar, Reena P. Borkakati and Gargi Sharma. 2020. Exploitation of Heterosis for Growth and Flowering Traits in kharif Marigold (Tagetes erecta L.). Int.J.Curr.Microbiol.App.Sci. 9(02): 808-820. doi: https://doi.org/10.20546/ijcmas.2020.902.098 